PERSPECTIVE

\title{
The Role of Professional Societies in Limiting Indication Creep
}

\author{
Kevin R. Riggs, M.D., M.P.H. ${ }^{1,2}$ and Peter A. Ubel, M.D., ${ }^{3,45}$ \\ 'Division of General Internal Medicine, Johns Hopkins University School of Medicine, Baltimore, MD, USA; ${ }^{2}$ Johns Hopkins Berman Institute \\ of Bioethics, Baltimore, MD, USA; ${ }^{3}$ Department of Medicine, School of Medicine, Duke University, Durham, NC, USA; ${ }^{4}$ Fuqua School of \\ Business, Duke University, Durham, NC, USA; ${ }^{5}$ Sanford School of Public Policy, Duke University, Durham, NC, USA.
}

New technology is a major driver of health care inflation. One contributor to this inflation is indication creep, the diffusion of interventions that have been proven beneficial in specific patient populations into untested broader populations who may be less likely to benefit. Professional societies sometimes promote indication creep, as we illustrate with the case of therapeutic hypothermia after cardiac arrest. Professional societies are in a unique position to limit indication creep. We propose that, at a minimum, professional societies should refrain from recommending new diagnostic and therapeutic technologies in their guidelines until they have been proven beneficial in the targeted populations. In some circumstances, professional societies could be more active in combatting indication creep, either recommending against expanded use of clinical interventions when evidence is lacking, or coordinating efforts to collect data in these broader populations.

KEY WORDS: indication creep; professional societies; guidelines; health care costs; ethics.

$\mathrm{J}$ Gen Intern Med 30(2):249-52

DOI: $10.1007 /$ s11606-014-2980-0

(c) Society of General Internal Medicine 2014

$\mathrm{R}$ ising health care costs are plaguing developed countries across the globe. As much as half of health care inflation can be attributed to new medical technologies. ${ }^{1}$ When new technologies provide significant benefits at reasonable cost, their influence on health care expenditures is easy to defend. But less defensible is the diffusion of interventions from populations where they were proven beneficial into untested populations where benefits are likely to be modest at best, a phenomenon known as indication creep. We argue that professional societies are in a unique position to limit indication creep by showing restraint in their practice guidelines. We propose that if potential recommendations represent indication creep, professional societies should refrain from issuing that

Received February 19, 2014

Revised June 11, 2014

Accepted July 9, 2014

Published online August 5, 2014 recommendation if it would be feasible to obtain high quality evidence that could eventually determine whether such a recommendation is supported or not.

\section{INDICATION CREEP AND ITS POTENTIAL HARMS}

The term indication creep was initially introduced to refer to the practice of pharmaceutical companies aggressively marketing medications for off-label indications, ${ }^{2}$ but recently has been used more broadly to refer to extrapolation beyond published clinical trial data to apply to individual patients. ${ }^{3}$ This is generally similar to the older term, excessive extrapolation. ${ }^{4}$ We wish to broaden and clarify the term to mean taking interventions that have proven benefits in specific patient populations and using them in untested patient populations, who are often less likely to benefit. This definition would include many off-label uses of pharmaceuticals, such as the use of atypical antipsychotics for delirium in hospitalized patients, but could also be applied to other therapeutics, such as the endovascular repair of abdominal aortic aneurysms that are smaller than recommended by trials and guidelines. ${ }^{5}$

Indication creep occurs when an intervention program to benefit patients with a specific health condition is either expanded to a broader patient population or expanded to a different health condition. As an example of population expansion, consider a new medication that is proven to benefit a relatively young and healthy population, but is then used in practice on patients who are older and have comorbid conditions. As an example of health condition expansion, consider a procedure proven to benefit patients who have experienced a prior stroke (secondary prevention), but which in practice is then prescribed for patients with risk factors for stroke (primary prevention).

Indication creep can harm individual patients. ${ }^{4}$ As interventions creep into new populations or different diseases, they typically do so in ways that lower the potential benefit: milder disease, which is less likely to cause problems, or sicker patients, who have higher competing risks of having problems regardless of whether they receive the intervention. Despite the lower potential benefit, the risks of the intervention will typically be the same or higher. ${ }^{4}$ This systematically pushes the balance away from aggregate health benefit towards harm. 
Indication creep can also cause societal harm. The use of such interventions can consume scarce resources, and thereby pull resources away from more beneficial activities. In addition, indication creep can contribute to rising health care costs, which can harm public health by reducing people's ability to access necessary medical care.

\section{AN EXAMPLE OF INDICATION CREEP: THERAPEUTIC HYPOTHERMIA}

To illustrate indication creep, consider the use of therapeutic hypothermia (TH) in survivors of cardiac arrest. The use of TH increased dramatically after the publication of two small randomized trials in 2002. ${ }^{6,7}$ The trials were conducted in patients experiencing out-of-hospital cardiac arrest with initial rhythms of either ventricular fibrillation (VF) or ventricular tachycardia (VT), and showed modest improvement in survival and neurologic status at hospital discharge and through six months. Costeffectiveness analysis even showed $\mathrm{TH}$ to be cost-effective in these patients. $^{8}$

However, use of TH has spread to other patient populations, including unwitnessed arrests, those with initial rhythms of pulseless electrical activity (PEA) and asystole, and in-hospital arrests. This indication creep has been encouraged by professional societies through practice guidelines. The 2005 American Heart Association ACLS guidelines gave a class IIb recommendation that $\mathrm{TH}$ "may be beneficial for patients with non-VF arrest out of hospital or for in-hospital arrest." Unfortunately, little data has been generated since then to support the expanded indications for TH. In fact, more recent randomized trials even call into question the effectiveness of $\mathrm{TH}$ in general. ${ }^{10}$

$\mathrm{TH}$ may be beneficial in patients beyond those shown in the initial trials, but such benefits are likely to be significantly smaller than in the populations tested in those trials. The patients in the trials had all of the best prognostic features of cardiac arrest - witnessed arrests, immediate resuscitations, and shockable rhythms - as confirmed by the fact that the control groups in the two trials experienced good outcomes $26-39 \%$ of the time. These favorable prognoses contrast sharply with the outcomes of cardiac arrest more generally. For example, in out-of-hospital PEA arrests, patients who survive to hospital admission only survive to discharge $16 \%$ of the time, and less than half of those have no or mild neurological deficit. ${ }^{11}$ The same relative benefit of therapeutic hypothermia, in the setting of a lower baseline probability of survival, necessarily leads to a smaller absolute treatment benefit.

\section{THE LIMITS OF OTHER ACTORS TO PREVENT INDICATION CREEP}

Given the decentralized structure of the U.S. healthcare system, there are few good options for efficiently preventing indication creep. The U.S. Food and Drug Administration (FDA) regulates the initial approval of medications and devices. Once approved, however, the FDA does not prevent use for off-label indications, the major driver of indication creep. Furthermore, some expensive technologies, such as $\mathrm{TH}$, are neither drugs nor devices, but "treatment strategies," and therefore lie outside the FDA's jurisdiction.

Third-party payers also have a difficult time in limiting indication creep. Private payers already resist covering marginally beneficial technologies to a degree, but they risk negative press and lawsuits when they deny care, as they did with bone marrow transplants for breast cancer in the 1980s. ${ }^{12}$ In the case of $\mathrm{TH}$, once respected professional societies like the American Heart Association make recommendations, thirdparty payers have more difficulty denying payment.

Medicare also has only a limited ability to restrain indication creep. Medicare is required to cover "reasonable and necessary" treatments; accordingly, it can deny coverage only for technologies that have not been shown to have any benefit. Once any benefit has been shown, Medicare is prohibited by law from explicitly considering cost or cost-effectiveness in its reimbursement decisions. ${ }^{13}$

\section{RATIONALE FOR PROFESSIONAL SOCIETIES' ABILITY TO PREVENT INDICATION CREEP}

There is no direct evidence that professional societies have an ability to prevent indication creep, but several factors suggest that they may. First, practice guidelines issued by professional societies can increase utilization of recommended interventions, ${ }^{14}$ which is one of the main objectives of formulating guidelines. When an intervention is proven to be beneficial, such as betablockers after myocardial infarction, practice guidelines are a mechanism by which that information can be quickly propagated. Second, once an intervention is in widespread use, it can be more difficult to assess its true effectiveness, particularly with randomized trials. For example, rituximab was initially used as secondline therapy for patients with idiopathic thrombocytopenic purpura (ITP) who could not tolerate the gold-standard treatment, splenectomy. However, its gradual widespread adoption into clinical practice as a first-line therapy (with the goal to delay or eliminate the need for splenectomy) has made it difficult to recruit patients for a randomized trial to determine its effectiveness compared to splenectomy. ${ }^{15}$ Recruiting patients for a trial of the expanded indications for TH would likely face the same difficulty. Therefore, professional societies may be able to slow indication creep through more careful recommendations in guidelines.

\section{TWO QUESTIONS TO CONSIDER}

In order to limit occurrences of indication creep, professional societies should ask two important questions before issuing 
recommendations. First, are the patients and disease factors similar to those where benefit has been shown? In other words, would this recommendation represent indication creep? We recognize that the majority of practices in clinical medicine today, even the majority of recommendations in practice guidelines, ${ }^{16,17}$ lack robust evidence of benefit, and we do not mean to suggest that any application of an intervention beyond inclusion criteria of a prior randomized trial should be considered indication creep. After all, even a well-conducted, randomized trial is essentially a "proof-of-concept," proving that an intervention can be efficacious under optimal conditions. Short of a "pragmatic trial," which is designed to prove effectiveness in real-world settings, ${ }^{18}$ the best evidence that can realistically be expected is one or more randomized trials followed by high quality observational data that supports effectiveness in actual clinical practice, which is likely to include patients who may not have met inclusion criteria for the initial trials. However, in the case of $\mathrm{TH}$, the recommendation for use in expanded patient populations (and arguably different conditions entirely) was made without even observational evidence of effectiveness.

Second, if the intervention being considered qualifies as indication creep, would it be feasible to obtain high quality evidence to support the recommendation (ideally by conducting a randomized trial)? Some practices may be so likely to be beneficial that it would be unethical to conduct the trials that would be needed to prove benefit. Others, like rituximab in ITP, may simply be too entrenched to realistically be able to recruit patients for a trial. However, with new technologies, a window for evaluation exists before the intervention becomes widespread. In such situations, professional societies should withhold recommendations (even weak recommendations) until more evidence is collected.

\section{WHAT ELSE PROFESSIONAL SOCIETIES CAN DO}

Expecting professional societies to simply "remain silent" and not make recommendations for certain interventions is admittedly a low bar to set for limiting indication creep - there is more they could potentially do. First, professional societies could make recommendations in their guidelines against use of an intervention because of a lack of evidence. However, this would be a dramatic change from current practice, and we are not necessarily advocating that individual physicians not utilize a potentially beneficial intervention simply because it is not "evidence-based". A reasonable compromise may be that guidelines recommend certain interventions be used only in the setting of a clinical trial, particularly when one is likely to be available. Second, professional societies could facilitate studies when their guideline committees have identified important gaps in evidence. Professional societies could help organize the study through a trial network, or even contribute to funding the study.

\section{CONCLUSION}

Indication creep poses a threat to quality patient care, as well as efforts to control health care spending. Other actors, such as the government and insurance companies, are limited in their ability to combat indication creep, which highlights the critical role that professional societies can play. Similar to the efforts of organized medicine to reduce waste through the Choosing Wisely campaign, ${ }^{19}$ professional societies should take the lead in preventing indication creep. By not recommending interventions that go beyond existing evidence where further trials to assess efficacy are feasible, and advocating for and facilitating important trials when they are needed, professional societies can display the restraint necessary to achieve the ultimate goal of improving quality while reducing costs.

\begin{abstract}
Acknowledgements: The authors gratefully acknowledge discussions on previous drafts with H. Gilbert Welch, Peter Neumann, and Victor Montori. Both authors were responsible for the content and drafting of the manuscript. Dr. Riggs' work on this manuscript was funded by NIH Grant T32HL007180-38 and a Hecht-Levi post-doctoral fellowship in bioethics. Dr. Ubel is supported in part by an Investigator Award in Health Policy from the Robert Wood Johnson Foundation. The funding sources had no role in the design and conduct of the study, analysis or interpretation of the data, and preparation or final approval of the manuscript prior to publication.
\end{abstract}

Conflict of Interest: The authors declare that they do not have a conflict of interest.

Corresponding Author: Kevin R. Riggs, M.D., M.P.H.; Division of General Internal Medicine, Johns Hopkins University School of Medicine, 2024 E. Monument Street, Room 2-604B, Baltimore, MD 21287, USA (e-mail: kriggs3@jhmi.edu).

\section{REFERENCES}

1. Smith S, Newhouse JP, Freeland MS. Income, insurance, and technology: why does health spending outpace economic growth? Health Aff. 2009;28:1276-1284.

2. Hebert PC, Stanbrook M. Indication creep: physician beware. CMAJ. 2007; $177: 697$.

3. Djulbegovic B, Paul A. From efficacy to effectiveness in the face of uncertainty: indication creep and prevention creep. JAMA. 2011;305:2005-2006.

4. Fisher ES, Welch HG. Avoiding the unintended consequences of growth in medical care: how might more be worse? JAMA. 1999;281:446-453.

5. Schanzer A, Greenberg RK, Hevelone N, et al. Predictors of abdominal aortic aneurysm sac enlargement after endovascular repair. Circulation. 2011;123:2848-2855.

6. Group THaCAS. Mild therapeutic hypothermia to improve the neurologic outcome after cardiac arrest. N Engl J Med. 2002;346:549-556.

7. Bernard SA, Gray TW, Buist MD, et al. Treatment of comatose survivors of out-of-hospital cardiac arrest with induced hypothermia. N Engl J Med. 2002;346:557-563. 
8. Merchant RM, Becker LB, Abella BS, Asch DA, Groeneveld PW. Costeffectiveness of therapeutic hypothermia after cardiac arrest. Circ Cardiovasc Qual Outcomes. 2009;2:421-428.

9. American Heart Association guidelines for cardiopulmonary resuscitation and emergency cardiovascular care. Part 7.5: postresuscitation support. Circulation. 2005; 112:84-8.

10. Nielsen $\mathbf{N}$, Wetterslev J, Cronberg T, et al. Targeted temperature management at 33 degrees $\mathrm{C}$ versus 36 degrees $\mathrm{C}$ after cardiac arrest. N Engl J Med. 2013;369:2197-2206.

11. Engdahl J, Bang A, Lindqvist J, Herlitz J. Factors affecting short-and long-term prognosis among 1069 patients with out-of-hospital cardiac arrest and pulseless electrical activity. Resuscitation. 2001;51:17-25.

12. Welch HG, Mogielnicki J. Presumed benefit: lessons from the American experience with marrow transplantation for breast cancer. BMJ. 2002;324:1088-1092.
13. Neumann PJ. Using cost-effectiveness analysis to improve health care: opportunities and barriers. New York: Oxford University Press; 2004.

14. Grol R, Grimshaw J. From best evidence to best practice: effective implementation of change in patients' care. Lancet. 2003;362:1225-1230.

15. Arnold DM, Heddle NM, Carruthers J, et al. A pilot randomized trial of adjuvant rituximab or placebo for nonsplenectomized patients with immune thrombocytopenia. Blood. 2012;119:1356-1362.

16. Tricoci P, Allen JM, Kramer JM, Califf RM, Smith SC Jr. Scientific evidence underlying the ACC/AHA clinical practice guidelines. JAMA. 2009;301:831-841.

17. Lee DH, Vielemeyer O. Analysis of overall level of evidence behind Infectious Diseases Society of America practice guidelines. Arch Intern Med. 2011;171:18-22.

18. Roland M, Torgerson DJ. What are pragmatic trials? BMJ. 1998;316:285.

19. Cassel CK, Guest JA. Choosing wisely. JAMA. 2012;307:1801-1802. 